\title{
Factors Associated with Common Mental Disorders among Women of Reproductive Age Group at Arbaminch Town, Southern Ethiopia: Cross Sectional Study
}

\author{
Yacob Abraham ${ }^{1 *}$, NiguseYigzaw ${ }^{2}$ and Fisseha Zewde ${ }^{3}$ \\ ${ }^{1}$ Department of Psychiatry, College of Medicine and Health Science, Hawasa University, Hawasa, Ethiopia \\ ${ }^{2}$ Department of Psychiatry, College of Medicine and Health Science, Gondar University, Gondar, Ethiopia
} ${ }^{3}$ Amanuel Mental Specialized Hospital, Addis Ababa, Ethiopia

\begin{abstract}
Background: Common mental disorders which include: Depression, Anxiety and Somatoform disorders make a significant contribution to the burden of disease and disability in low and middle income countries. Women are more exposed and affected by common mental disorders than men as reported by different studies from developing and developed countries. The condition is worst in low income countries. Therefore the aim of this study was to assess prevalence and associated factors of Common Mental Disorders among Women of reproductive age group at Arbaminch Town, southern Ethiopia.

Methods: Community based cross sectional study was conducted from April 20 to May 20, 2014 in Arbaminch town. The data was collected by using interviewer administered questionnaire. The study was conducted among 845 women of reproductive age group among Arbaminch town residents; in which household was taken as a sampling unit. Common mental disorder was assessed using the self-reporting questionnaire. Bivariate and Multivariate logistic regression analysis was carried out to identify factors associated with common mental disorders and P-value $<0.05$ was taken as statistically significant.

Results: A total of 845 questionnaires were distributed and 835 were available for analysis giving a response rate of $98.8 \%$. The prevalence of common mental disorders was found to be $31 \%$. Family history of mental illness(AOR=4.36;95\% Cl:1.68,11.34)., chronic medical illness (AOR=2.96,95\% Cl:1.96,4.47) and early age at first marriage(AOR=1.91;95\% Cl:1.32,2.77) were significantly associated factors with common mental disorders.

Conclusion: The prevalence of Common mental disorders among women of reproductive age group population at Arbaminch town was significantly high. Common mental disorders were significantly associated with family history of mental illness, chronic medical illness and early age at first marriage in the town. Therefore proper screening for common mental disorders among reproductive age group is crucial and mother during ANC follow up should be screened for common mental disorders. Particularly emphasis should be given for those who have family history of mental illness, women with chronic medical illness and women married before age of 18 .
\end{abstract}

Keywords: Common mental disorder; Reproductive age group

Abbreviations: AOR: Adjusted Odds Ratio; IRB: Institutional Review Board; CMDs: Common Mental Disorders; SNNP: Southern Nation Nationality and People of Ethiopia; SRQ: Self-Reporting Questionnaire; SPSS: Statistical Package for Social Sciences; ART: Antiretroviral Therapy

\section{Introduction}

Common Mental disorders which includes: Depression, Anxiety and Somatoform disorders make a significant contribution to the burden of disease and disability in low and middle income countries [1].

The concept of CMDs characterizes cases that present nonpsychotic symptoms, such as insomnia, fatigue, nervousness, headache, depressive symptoms, irritability, forgetfulness, difficulty concentration, and non-specific symptoms that lead to functional incapacity but do not meet the requirement of diagnosis listed in the DSM-IV [2]. In regard of gender, women are more likely to be adversely affected by mental disorders than men. Community based cross-sectional study done in Turk on prevalence of any mental disorders among women of 15-49 years was $25.9 \%$. The study stated that women were at heightened risk for common mental disorders: a female to male sex ratio of 1.5 to $2.0[3]$.

Studies done among middle-aged Finnish and British public sector employees showed that the overall age-adjusted prevalence of common mental disorders was $26 \%$ among women and $23 \%$ among men in the Helsinki cohort [4]. Study done in rural Kenya reported prevalence of CMDs was $10.8 \%$, with no gender deference [5].

The result of Study done in urban Tanzanian mothers of age 1548 suggested that more than 1 in 4 mothers were at risk of CMDs (28.8\%) [6]. Cross-sectional data from the Young Lives (YL) study with information across 234 communities in Peru, Ethiopia, Vietnam and Andhra Pradesh (India) reported that twenty-one percent of mothers in the sample from Vietnam were probable cases of CMD, compared to around $30 \%$ in the other three countries (Peru, Ethiopia and India) [7].

*Corresponding author: Yacob Abraham, Department of Psychiatry, College of Medicine and Health Science, Hawasa University, Hawasa, Ethiopia, Tel: 2511911 99-96-24; E-mail: yacobabraham12@gmail.com.

Received: October 30, 2017; Accepted: November 28, 2017; Published: December 08, 2017

Citation: Abraham Y, Yigzaw N, Zewde F (2018) Factors Associated with Common Mental Disorders among Women of Reproductive Age Group at Arbaminch Town, Southern Ethiopia: Cross Sectional Study. J Psychiatry 21: 436. doi:10.4172/23785756.1000436

Copyright: @ 2018 Abraham Y, et al. This is an open-access article distributed under the terms of the Creative Commons Attribution License, which permits unrestricted use, distribution, and reproduction in any medium, provided the original author and source are credited 
Cross-sectional study conducted among working adults in Addis Ababa, Ethiopia showed that the prevalence of mental distress (common mental disorder) was $17.7 \%$ (25.9\% in women and in men $12.4 \%)$. The study used SRQ-20 tool at cut point 6 . Women were more likely to have higher prevalence of CMD compared with men across all age groups. The odds of mental disorders were 2.47 fold higher among women as compared to men. In the study investigators showed that mental health problems; particularly depression, anxiety and somatic complaints affect women to greater extent than men across divers societies and social contexts [8].

Early marriage was associated factor of gender disadvantages as indicated by the following studies: community based study in India among women of 18-50 years stated that being married during adolescence was significantly associated with CMDs [9]. Community based cross-sectional study in Pakistan among women of 18-35 years showed that there was significant association of being married at earlier age $(<20$ year) and CMD [10]. Early pregnancy poses great health risks for young women and these risks are exacerbated by poverty and inadequate access to maternal and child health services [11]

Findings based on Mental Health Supplement of the German National Health interview and Examination showed that separated, divorced or widowed women had higher prevalence of any mental disorders than married women [12]

Cross -sectional study conducted in Santiago, Chile reported that age was associated with the prevalence of CMDs. In the study highest prevalence was found among those individuals aged 25-39 years and the lowest in the oldest age group(40-54 years) [13]

In cross-sectional study conducted in Butajira, Ethiopia, marital status showed significant positive association with mental distress, single groups suffered from mental distress to lesser extent in all age groups than married, widowed and divorced groups. The study also reported significance association among illiteracy, poverty, older age group and small family size and mental distress [14]

CMDs are associated with physical health conditions, particularly those with chronic medical conditions, the co-morbidity of depression and chronic diseases dramatically increasing the risk of poor health outcomes. Organic diseases like cerebra-vascular diseases, nervous system diseases, endocrine diseases and chronic illnesses such as epilepsy were associated with mental illnesses as reported by studies in USA and South Africa $[15,16]$.

Common mental disorders can lead to high social, economic and individual costs. Since such disorders account one third of the days missed at work and fifth of all PHC appointment.

CMDs are known to produce disability and increase health costs. So failure to address CMDs causes, major social and financial burdens for families, friends and employers [17].

Although few available studies indicate the relevance of mental disorders, data on the mental health status of population are still scarce, causing a lack of information about psychiatric morbidity.

In Ethiopia there was few epidemiological data on the prevalence and associated factors of CMDs. Studies showed that women are more vulnerable than men to have CMDs. Therefore this study was aimed to assess prevalence of common mental disorder and associated factors of common mental disorders among reproductive age group women in Arbaminch town, Gamo-Gofa Zone, SNNPR, Ethiopia. The findings of this study would help in developing evidence based mental health promotion and disease prevention programs that are relevant to the study subjects and make some contributions to improve mental health services and create insight for policy makers for appropriate intervention strategies (Figure 1).

\section{Methods}

\section{Study setting and population}

Community based cross sectional study was carried out from April 20 to May 20, 2014 in Arbaminch town, Gamo-Gofa Zone, South Nation Nationalities regional state of Ethiopia. All selected women aged 15 to 49 years who lived in the study area for 6 months or more, were included in the sample. Subjects who were seriously ill during the data collection were excluded. Estimated number of households of the town based on the 2007 census conversion factor was about 20498, which comprised of an estimated women of childbearing age 24305 (15-49 years). The final sample size was 845 . A multi stage sampling Technique was used. At stage one from the 11 Kebeles 3 was selected randomly using lottery method. Then individual households in the chosen Kebeles were selected using a systematic sampling technique after identifying an initial starting household by use of a random number. Women in the selected household were further selected and interviewed.

\section{Data collection}

Data was collected by trained nurses using face to face interviewer The data collection instrument had five parts. The first part was sociodemographic information. The second part of the questionnaire was a Self-Reporting Questionnaire (SRQ20). Self-reporting questionnaire (SRQ) was used to assess common mental disorders. The SRQ was developed by WHO within the framework of a collaborative study on Strategies for Extending Mental Health Care [18]. The SRQ has previously been translated into Amharic and validated in Ethiopia [19], and it has been used for community surveys. The cut-off point for the tool was presence of 6 or more symptoms and which was used by previous studies. Third part of the questionnaire was about behavioral factors, which comprised of history of substance use in their life time and current use. The fourth part contained questions related to age at first marriage. The final part of the questionnaire was about clinical factors: presence of chronic medical disease and family history of mental illness.

\section{Common mental disorder}

In this study, women who were found to have symptoms of the 20 SRQ 20 questions at cut point of 6 or more in the last 4 weeks were considered as having common mental disorders.

\section{Data processing and analyses}

Data were analyzed using SPSS version 20. Multivariate and bivariate logistic regression analysis was done to see the association of each independent variable with the outcome variable. Potential confounders' variables were entered into binary logistic regression model to identify the effect of each independent variable with the outcome variables. A p-value of less than 0.05 was considered statistically significant.

\section{Result}

A total of 845 participants were enrolled on the study with a response rate of $98.8 \%$. The median age of respondents was 30 years with inter quartile range of 13 . Most of the participants were Gamo by ethnicity (52.6\%). Most of the participants $55.1 \%$ were married. Most of the participants had primary educational level (33.4\%). The median of 
Citation: Abraham Y, Yigzaw N, Zewde F (2018) Factors Associated with Common Mental Disorders among Women of Reproductive Age Group at Arbaminch Town, Southern Ethiopia: Cross Sectional Study. J Psychiatry 21: 436. doi:10.4172/2378-5756.1000436

Page 3 of 6

age at first marriage was 18 years with interquartile range of 4 .

From married women $58.3 \%$ were reported to get their first marriage at age of 18 years and above and $43.2 \%$ at age of 17 years and below (Tables 1 to 4 ).

\section{Prevalence of common mental disorder}

The prevalence of common mental disorder among reproductive age group women was found to be $31 \%$ with $95 \%$ CI $(28,34)$ (Figure 2 ).

\section{Associated factors of common mental disorders}

Women with family history of mental illness are 4.36 times more likely to develop Common mental illness when we compare with women with no family history of mental illness(AOR $=4.36 ; 95 \% \mathrm{CI}: 1.68,11.34$ ). Being early married at first marriage are 2 times more likely to develop common mental disorders when we compared to those lately married women (AOR=1.91;95\% CI:1.32,2.77). Women with chronic medical illness are 2.96 times more likely to develop common mental disorders when compared with than women with no chronic medical illness $(\mathrm{AOR}=2.96 ; 95 \% \mathrm{CI}: 1.96,4.47)$ (Table 5).

\section{Discussion}

Community based cross-sectional study was conducted to assess the prevalence and associated factors of common mental disorder among women of reproductive age group at Arbaminch town. The findings showed the prevalence of common mental disorders among women of reproductive age group at Arbaminch town was 31\%.

The prevalence rate in this study was higher than the Community based cross-sectional study done in Turk among women of reproductive age group which was $25.9 \%$ [11]. The difference may be due to difference in study population in culture and tools used. Studies done among adult middle-aged Finnish and British public sector employees showed that the overall age-adjusted prevalence of common mental disorders was $26 \%$ among women in the Helsinki cohort. The corresponding figure was $29 \%$ in the London Cohort [12] which was lower than the current study. This study prevalence was about 3 times higher than study done among adults in rural Kenya which reported prevalence of CMDs $10.8 \%$, with no gender deference [13] again the difference can be attributed to difference in study population in culture, age distribution and instrument used. The prevalence rates found were similar compared to study conducted in Tanzanian mothers of reproductive age group. The prevalence of CMDs among Tanzanian mothers of age 15-48 was $28.8 \%$

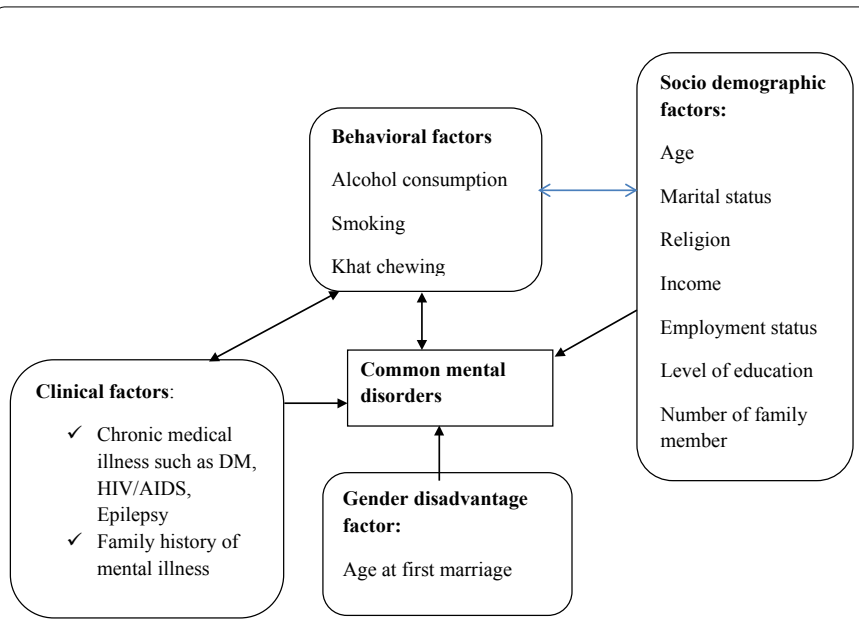

Figure 1: Conceptual framework derived from literatures.

\begin{tabular}{|c|c|c|c|}
\hline Variables & Category & Frequency & Percent (\%) \\
\hline Age (in years) & $\begin{array}{c}15-19 \\
20-24 \\
24-29 \\
30-34 \\
\geq 35\end{array}$ & $\begin{array}{c}135 \\
97 \\
132 \\
193 \\
278\end{array}$ & $\begin{array}{l}16.2 \\
11.6 \\
15.8 \\
23.1 \\
33.3\end{array}$ \\
\hline $\begin{array}{l}\text { Educational } \\
\text { status }\end{array}$ & $\begin{array}{c}\text { Unable to read and write } \\
\text { Primary } \\
\text { High school } \\
\text { College and above }\end{array}$ & $\begin{array}{l}111 \\
279 \\
278 \\
167\end{array}$ & $\begin{array}{l}13.3 \\
33.4 \\
33.3 \\
20.0\end{array}$ \\
\hline Job & $\begin{array}{c}\text { Merchant } \\
\text { Civil servant } \\
\text { Private employee } \\
\text { Day labor } \\
\text { Student } \\
\text { Unemployed }\end{array}$ & $\begin{array}{c}62 \\
82 \\
136 \\
117 \\
179 \\
259\end{array}$ & $\begin{array}{c}7.4 \\
9.8 \\
16.3 \\
14.0 \\
21.4 \\
31.0\end{array}$ \\
\hline Marital status & $\begin{array}{l}\text { Never married } \\
\text { Married } \\
\text { Divorced } \\
\text { Widowed }\end{array}$ & $\begin{array}{c}257 \\
481 \\
56 \\
41\end{array}$ & $\begin{array}{c}30.8 \\
57.6 \\
6.7 \\
4.9\end{array}$ \\
\hline Ethnicity & $\begin{array}{l}\text { 1. Gamo } \\
\text { 2. Gofa } \\
\text { 3. Amhara } \\
\text { 4. Other }\end{array}$ & $\begin{array}{c}439 \\
225 \\
128 \\
43\end{array}$ & $\begin{array}{c}52.6 \\
26.9 \\
15.3 \\
5.1\end{array}$ \\
\hline Religion & $\begin{array}{l}\text { 1. Protestant } \\
\text { 2. Orthodox } \\
\text { 3. Muslim } \\
\text { 4. Catholic }\end{array}$ & $\begin{array}{c}331 \\
417 \\
57 \\
30\end{array}$ & $\begin{array}{c}39.6 \\
49.9 \\
6.8 \\
3.6\end{array}$ \\
\hline Family size & $\begin{array}{l}1-2 \\
3-5 \\
\geq 6\end{array}$ & $\begin{array}{l}144 \\
490 \\
201\end{array}$ & $\begin{array}{l}17.2 \\
58.7 \\
24.1\end{array}$ \\
\hline Monthly income & $\begin{array}{c}<750 \text { ETB } \\
750-1199 \text { ETB } \\
\geq 1200 \text { ETB }\end{array}$ & $\begin{array}{c}742 \\
70 \\
23\end{array}$ & $\begin{array}{c}88.9 \\
8.4 \\
2.8\end{array}$ \\
\hline
\end{tabular}

Table 1: Socio demographic characteristics of the study participants in Arbaminch town, 2014.

\begin{tabular}{|c|c|c|}
\hline Substance ever use & Frequency & Percent \\
\hline Never ever use & 409 & 49 \\
\hline Alcohol ever use & 294 & 35.2 \\
\hline Khat ever use & 36 & 4.3 \\
\hline Alcohol and khat ever use & 96 & 11.5 \\
\hline Substance current use & & \\
\hline Not used at all & 523 & 62.6 \\
\hline Alcohol & 241 & 28.9 \\
\hline Khat & 32 & 3.8 \\
\hline Alcohol and khat & 39 & 4.7 \\
\hline
\end{tabular}

Table 2: Substance use characteristics of participant in Arbaminch town, 2014.

\begin{tabular}{|c|c|c|}
\hline Age at first marriage & Frequency & Percent (\%) \\
\hline Not ever married & 252 & 30.2 \\
\hline$\leq 17$ years & 252 & 30.2 \\
\hline$\geq 18$ & 33 & 39.6 \\
\hline
\end{tabular}

Table 3: Gender disadvantage related characteristics of participant in Arbaminch 2014.

\begin{tabular}{|c|c|c|c|}
\hline Variables & Category & Frequency & Percent (\%) \\
\hline $\begin{array}{c}\text { Chronic medical } \\
\text { illness }\end{array}$ & Yes & 139 & 16.6 \\
\cline { 2 - 4 } & No & 696 & 83.4 \\
\hline \multirow{2}{*}{$\begin{array}{c}\text { Family history of } \\
\text { mental illness }\end{array}$} & Yes & 22 & 2.6 \\
\cline { 2 - 4 } & No & 813 & 97.4 \\
\hline
\end{tabular}

Table 4: Clinical characteristics of participants in Arbaminch, 2014. 
Citation: Abraham Y, Yigzaw N, Zewde F (2018) Factors Associated with Common Mental Disorders among Women of Reproductive Age Group at Arbaminch Town, Southern Ethiopia: Cross Sectional Study. J Psychiatry 21: 436. doi:10.4172/2378-5756.1000436

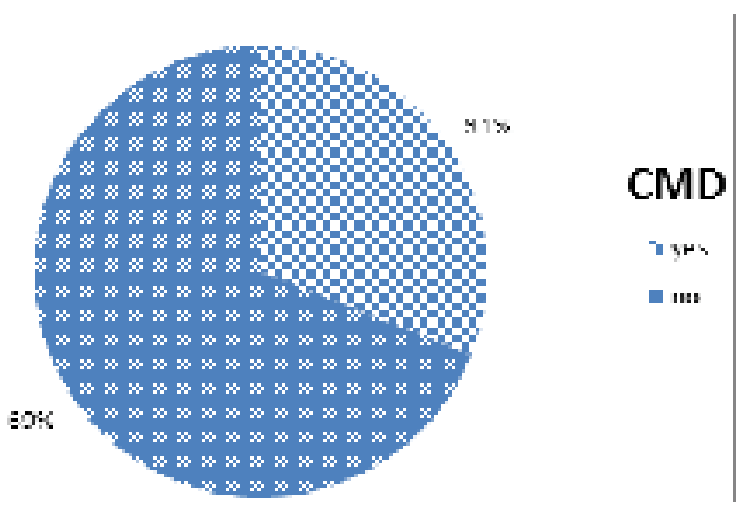

Figure 2: Prevalence of common mental disorder among women of reproductive age group at Arbaminch town, 2014

\begin{tabular}{|c|c|c|c|c|}
\hline \multirow[t]{2}{*}{ Variables } & \multicolumn{2}{|c|}{ CMDs } & \multirow[t]{2}{*}{ Crude odds ratio, $\mathrm{CI}$} & \multirow[t]{2}{*}{ Adjusted odds ratio, $\mathrm{Cl}$} \\
\hline & Yes & No & & \\
\hline \multicolumn{5}{|l|}{ Status of job } \\
\hline Merchant & 22 & 40 & 1 & 1 \\
\hline Civil servant & 22 & 60 & $0.66(0.32,1.36)$ & $0.68(0.31,1.50)$ \\
\hline Private employee & 39 & 97 & $0.73(0.38,1.38)$ & $0.95(0.47,1.93)$ \\
\hline Day labor & 50 & 67 & $1.35(0.71,2.56)$ & $1.17(0.58,2.37)$ \\
\hline Student & 47 & 132 & $0.64(0.34,1.20)$ & $1.09(0.51,2.31)$ \\
\hline Unemployed & 79 & 180 & $0.79(0.44,1.43)$ & $0.62(0.32,1.19)$ \\
\hline \multicolumn{5}{|l|}{ Marital status } \\
\hline Never married & 56 & 201 & 1 & 1 \\
\hline Married & 159 & 322 & $1.77(1.24,2.52)$ & $1.76(0.72,4.30)$ \\
\hline Divorced & 23 & 33 & $2.50(1.36,4.60)$ & $1.98(0.69,5.65)$ \\
\hline Widowed & 21 & 20 & $3.76(1.90,7.44)$ & $2.28(0.72,7.12)$ \\
\hline \multicolumn{5}{|l|}{ Educational level } \\
\hline Unable to read and write & 50 & 61 & $1.97(1.19,3.25)$ & $1.06(0.52,2.12)$ \\
\hline Primary & 90 & 189 & $1.14(0.75,1.74)$ & $0.78(0.43,1.39)$ \\
\hline High school & 70 & 208 & $0.81(0.52,1.24)$ & $0.71(0.43,1.18)$ \\
\hline College and above & 49 & 118 & 1 & 1 \\
\hline \multicolumn{5}{|l|}{ Monthly income } \\
\hline$<750$ ETB & 239 & 504 & $1.07(0.43,2.65)$ & $0.791(0.28,2.21)$ \\
\hline 750-1199ETB & 14 & 56 & $0.57(0.19,1.65)$ & $0.42(0.13,1.36)$ \\
\hline$\geq 1200 \mathrm{ETB}$ & 7 & 16 & 1 & 1 \\
\hline \multicolumn{5}{|l|}{ Age at first marriage } \\
\hline Not married & 55 & 197 & $0.73(0.50,1.08)$ & $0.92(0.61,1.39)$ \\
\hline$\leq 17$ years & 113 & 139 & $2.14(1.51,3.03)$ & $1.91^{* *}(1.32,2.77)$ \\
\hline$\geq 18$ years & 91 & 240 & 1 & 1 \\
\hline \multicolumn{5}{|l|}{ Family history of mental illness } \\
\hline No & 245 & 568 & 1 & 1 \\
\hline Yes & 14 & 8 & $4.05(1.68,9.79)$ & $4.36^{* *}(1.68,11.34)$ \\
\hline \multicolumn{5}{|l|}{ Chronic medical illness } \\
\hline No & 183 & 513 & 1 & 1 \\
\hline Yes & 76 & 63 & $3.32(2.32,4.91)$ & $2.96^{\star *}(1.96,4.47)$ \\
\hline
\end{tabular}

Note: ${ }^{* *}=$ shows statistically significant variables

Table 5: Multivariate and bivariate analysis of associated factors of common mental disorder among women of reproductive age group at Arbaminch town, 2014.

[14]. The prevalence of Young live study report rates among women in 3 countries (Ethiopia, Peru and India) is comparable to this finding, where the prevalence of CMDs was $30 \%$ in these countries [15]. When comparing this prevalence with study conducted among working adults in Addis Ababa, Ethiopia which was $12.4 \%$ in men and $25.9 \%$ in women it was higher. The difference may be due to difference in study subjects in age, educational level and employment status that may be attributing factors for this study to be higher than previous cross-sectional study [16]. So, the finding confirmed higher prevalence of common mental disorder among reproductive age group women as compared to adult 
men and women in Ethiopia.

This finding showed that women married during adolescent age at first marriage ( $\leq 17$ years) were found almost twice as likely report CMDs as compared with women married their first marriage age at 18 or greater years.

This finding showing significant association of being married earlier and CMD was consistent with previous community based crosssectional studies in India and Pakistan $[18,19]$.

As investigators noticed early marriage typically coincides with childbearing at young age. Early pregnancy poses great health risks for young women and, if she carries the pregnancy to term, for her infant; these risks were exacerbated by poverty and inadequate access to maternal and child health services. Early age at first marriage was associated higher probability of divorce and separation. In turn marriage dissolution creates social and economic challenges for women who, as single parents often assume full responsibility for dependent family members.

In this study socio demographic factor such as marital status, educational status were not significantly associated with common mental disorder but these were significant factors in previous literatures in Santiago, Chile and Butajira, Ethiopia. The difference may be due to study population difference in culture and composition for study done in Santiago and study population difference in age, composition for study done in Ethiopia.

The result of this study also had shown significantly strong association of having history of mental illness in the family and CMDs. This finding was consistent with previous cross sectional studies in Harumaya and Addis Ababa, Ethiopia which suggested genetic factors as possible cause for increased risk of common mental illness in family with positive history of mental illness. In this study family history of mental illness and common mental disorders association could be possibly due to genetic factors and psychosocial stressor produced by burden of mental illness in the family may contribute to increase like hood of common mental disorders to individual family membranes.

In present study having chronic medical illness was significantly associated with prevalence of CMDs. Chronic illness such as cardiovascular disease, DM, HPN and others like epilepsy, leprosy and TB had strong significant association with CMDs when compared with having no chronic illness. The finding was consistent with previous studies in the USA and South Africa.

Situations related with chronic physical illness like long time and continuous medication, medication side effect, expense of medication, feeling of insecure due to not being cured are long time stressor and other psychosocial stressors may be associated with having chronic illness are stigmatization, loss of employment or inability to work effectively can cause financial disadvantage and can predispose or precipitated common mental disorders.

The finding of this study should be interpreted with some limitation. Being a cross-sectional study, causal inference cannot be made between CMDs and independent variables. Administering the questionnaire through face to face interview may have resulted socially desirable response.

\section{Conclusion}

The prevalence of Common mental disorders among women of reproductive age group population at Arbaminch town was found significantly high. Common mental disorders were significantly associated with family history of mental illness, chronic medical illness and early age at first marriage in the town.

Therefore proper screening for common mental disorders among reproductive age group is crucial to prevent and detect and treat early so as to prevent from severe mental illness thereby preventing functional impairments like social, occupational. Special emphasis should be given for those who have family history of mental illness, women with chronic medical illness and those married before age 18 .

\section{Declaration}

Ethical Consideration and Consent to participate: Ethical clearance was obtained from Ethical review board of university of Gondar and Emanuel specialized mental hospital; in order to obtain permission letter Arbaminch town health unit was contacted. Ethical review board approved the method of data collection and consent. Then the selected women (15 years to 49 of age ) from each household were informed about the purpose of the study, the importance of their participation, withdraw at any time and written consent was obtained to collect and publish the data prior to data collection. And for those selected study subjects from 15 up to 18 years of age assent form was prepared and written assent was obtained prior data collection. Privacy and confidentiality of information given by each respondent was kept properly and names were not recorded. Mentally ill cases found during the study period were linked to Arbaminch general hospital for further investigation and treatment.

\section{Consent for Publication}

Not applicable.

\section{Competing Interests}

The authors declare that they have no competing interests.

\section{Authors' Contributions}

Yacob Abraham conceived the study and involved in the study design, reviewed the article, analysis, report writing and drafted the manuscript. NiguseYigzaw and Fisseha Zewde were involved in the study design, analysis and drafted the manuscript. All authors read and approved the final manuscript.

\section{Acknowledgement}

The authors acknowledge Emanuel Mental Specialized Hospital and University of Gondar for funding the study. The authors also would like to thank the respective study institutions and the study participants for their cooperation in providing al necessary information

\section{References}

1. Lopez DA, Ezzati M, Jamison TD, Murray JLC, Mathers CD (2006) Global and regional burden of disease and risk factors, 2001: systematic analysis of population health data. Lancet 367: 1747-1757.

2. Fonseca MLG, Vasconcelos EM, Guimarães MBL (2008) Diffuse suffering and common mental disorders: a bibliographic review. Rev APS 11: 285-294.

3. Simsek Z, Ak D, Altindag A, Günes M (2008) Prevalence and predictors of mental disorders among women in Sanliurfa, Southeastern Turkey. J Public Health 30: 487-449.

4. Laaksonen E, Martikainen P, Lahelma E, Lallukka T, Rahkonen O, et al. (2007) Socio economic circumstances and common mental disorders among Finnish and British public sector employees: evidence from the Helsinki Health Study and the Whitehall II Study. Int J Epidemiol 36: 776-786.

5. Jenkins R, Njenga F, Okonji M, Kigamwa P, Baraza M, et al. (2012) Prevalence of common mental disorders in a rural district of Kenya, and socio-demographic risk factors. Int J Environ Res Public Health 9: 1810-1811.

6. Jacqueline G, Uriyo A, Mark S, Sia EM, Babill SP (2013) Prevalence and correlates of common mental disorders among mothers of young children in Kilimanjaro region of Tanzania. PLOS ONE 8. 
Citation: Abraham Y, Yigzaw N, Zewde F (2018) Factors Associated with Common Mental Disorders among Women of Reproductive Age Group at Arbaminch Town, Southern Ethiopia: Cross Sectional Study. J Psychiatry 21: 436. doi:10.4172/2378-5756.1000436

7. De Silva MJ, Huttly SR, Harpham T, Kenward MG (2007) Social capital and mental health: A comparative analysis of four low income countries. Soc Sci Med 64: 5-20.

8. Gelaye B, Seblewengel L, Negussie D, Yonas B, Markos T, et al. (2012) Prevalence and Correlates of Mental Distress among Working Adults in Ethiopia. Clin Pract Epidemiol Ment Health 8: 126-133.

9. Patel V, Kirkwood BR, Pednekar S, Pereira B, Barros P, et al. (2006) Gender disadvantage and reproductive health risk factors for common mental disorders in women. Arch Gen Psychiatry 63: 404-413.

10. Farah Q, Murad MK, Girmay M, Martin P (2011) Male gender preference, female gender disadvantage as risk factors for psychological morbidity in Pakistani women of childbearing age - a life course perspective. BMC Public Health 11: 745

11. Sanpra SS (1996) International FP perspectives.

12. Jacobi F, Klose M (2004) Can gender differences in the prevalence of mental disorders be explained by socio-demographic factors? Arch Women's Ment Health 7: 133-148.

13. Araya R, Rojas G, Fritsch R, Acuña J, Lewis G (2001) Common mental disorders in Santiago, Chile: Prevalence and socio-demographic correlates. $\mathrm{Br}$

\section{J Psychiatry 178: 228-233.}

14. Alem A, Kebede D, Woldesemiat G, Jacobsson L, Kullgren G (1999) The prevalence and socio demographic correlates of mental distress in Butajira, Ethiopia. Acta Psychiatrica Scandinavica Suppl 100: 48-55.

15. Roy-Byrne PP, Davidson KW, Kessler RC, Asmundson GJ, Goodwin RD, et al (2008) Anxiety disorders and comorbid medical illness. Gen Hosp Psychiatry 30: 208-225.

16. Landon M, Smit J, Roux Liezel Le, Parker S, Stein Dan J, et al. (2008) Common mental disorders among HIV-infected individuals in South Africa: Prevalence, predictors, and validation of brief psychiatric rating scales. AIDS Patient Care and STDs 22: 147-158.

17. Fortes S, Luiz ABV, Lopes CS (2008) Nosological profile and prevalence of common mental disorders of patients seen at the Family Health Program (FHP) units in Petrópolis, Rio de Janeiro. Rev Bras Psiquiatr 30: 32-37.

18. M Beusenberg, Orley J (1994)A user's guide to the Self Reporting Questionnaire (SRQ). WHO, Geneva.

19. Mulatu MS (1995) Prevalence and risk factors of psychopathology in Ethiopian children. J Am Acad Child Adolesc Psychiatry 34: 100-109. 